\title{
How low is the risk of mild COVID-19 illness? Cardiovascular Imaging for Longitudinal Sequelae
}

\author{
Miguel Ayala-Leon ${ }^{1}$, Rosa Tzompantzi-Flores ${ }^{2}$, Milton González Henao ${ }^{1}$, Jaime \\ Hernandez-Montfort ${ }^{3}$, Cosnuelo Orihuela Sandoval ${ }^{4}$, and Luis Bérmudez Pérez ${ }^{5}$ \\ ${ }^{1}$ Universidad Popular Autonoma del Estado de Puebla \\ ${ }^{2}$ Benemerita Universidad Autonoma de Puebla \\ ${ }^{3}$ Cleveland Clinic \\ ${ }^{4}$ Instituto Nacional de Ciencias Médicas y Nutrición Salvador Zubiran \\ ${ }^{5}$ Hospital Sociedad Española de Beneficencia de Puebla
}

July 24, 2020

\begin{abstract}
Report on the relevance of the stress echocardiography after mild COVID-19 illness; this is significant for targeting specific therapies and addressing progression or regression of abnormalities in the cardiovascular system.
\end{abstract}

To the editor:

With the appearance of the new SARS-COV2 virus, additional challenges are being imposed to the cardiovascular imaging community after resolution of acute COVID-19 illness resulting in specific pathophysiologic mechanism that while acutely damage the lung parenchyma (i.e. diffuse alveolar hemorrhage, intra-alveolar organizing fibrin) might chronically impact the cardiopulmonary system (1).

We report the case of a 26-year-old man with prior mild COVID-19 illness, with negative serology test (IgG+ / IgM-) and lung tomography without fibrosis neither pulmonary embolism, who while in cardiopulmonary rehabilitation referred due to premature ventricular complexes associated with mild dyspnea on exertion. Decision was made to proceed with stress echocardiography are requested to evaluate the right ventricle (RV) contractile reserve. At rest, we encountered normal RV function with a low probability of pulmonary hypertension with systolic pulmonary artery pressure (SPAP) $32 \mathrm{mmHg}$ followed by stress suggestive of exercise-induced pulmonary hypertension (SPAP $60 \mathrm{mmHg}$ ) (Figure 1). Comparing other variables (rest vs. stress) showed differences in the strain of RV free wall (32 vs 38), a delta on TAPSE of 12: (20 vs 32), a delta on S wave of 10: (13 vs 23) suggestive of good contractile RV reserve (2). Evaluation of right ventricle arterial coupling (surrogate of right ventricular stiffness) was adequate at 0.35 ; however, the shortening of the pulmonary artery during exercise was reduced by - 60, which translates into increased pulmonary vascular resistance. The pulmonary vascular reserve in this patient was decreased, which may indicate a subclinical phase of pulmonary vascular disease.

Assessment of left ventricular function was normal, with an adequate increment of the ejection fraction during stress (60\% vs $72 \%$ ) and a delta on $\mathrm{e}^{\prime}$ wave of 7 (4 vs 11) (3). 
These findings highlight the need for all patients recovering from COVID-19, even though present mild pneumonia, to undergo to risk stratification with stress echocardiographic evaluation to diagnose and monitor early signs of pulmonary hypertension as this presented case. Furthermore opens the dialogue for targeting specific therapies and addressing progression or regression of abnormalities associated with the right heart unit. Importantly, as we enter a new era in the longitudinal cardiovascular evaluation in the midst of a pandemic, our case highlights the need for further studying the role of exercise echocardiography among patients recovering from COVID-19 illness.

1. Xu, Z., Shi, L., Wang, Y., Zhang, J., Huang, L., Zhang, C., .. \& T Tai, Y. (2020). Pathological findings of COVID-19 associated with acute respiratory distress syndrome. The Lancet respiratory medicine , 8 (4), 420-422.

2. D'Alto, M., Pavelescu, A., Argiento, P., Romeo, E., Correra, A., Di Marco, G. M., ... \& Naeije, R. (2017). Echocardiographic assessment of right ventricular contractile reserve in healthy subjects. Echocardiography, 34 (1), 61-68.

3. Larsen, A. H., Clemmensen, T. S., Wiggers, H., \& Poulsen, S. H. (2018). Left ventricular myocardial contractile reserve during exercise stress in healthy adults: a two-dimensional speckle-tracking echocardiographic study. Journal of the American Society of Echocardiography , 31 (10), 1116-1126.
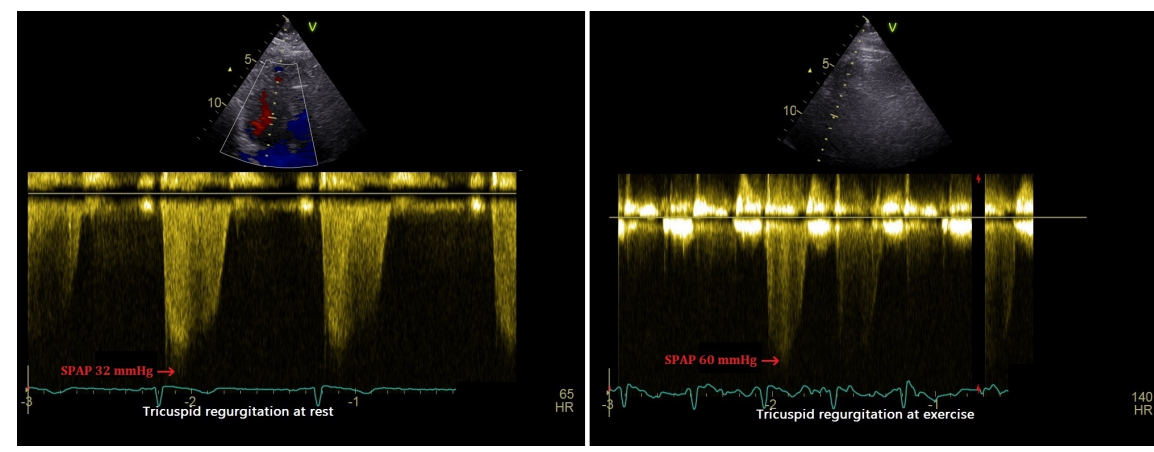